\title{
Persistent parochialism: trust and exclusion in ethnic networks
}

\author{
Samuel Bowles a , Herbert Gintis ${ }^{b, *}$ \\ a Santa Fe Institute, University of Siena, Italy \\ b Santa Fe Institute, Columbia University, USA \\ Received 7 January 2003; accepted 2 June 2003 \\ Available online 28 May 2004
}

\begin{abstract}
Decentralized groups such as close knit residential neighborhoods and ethnically linked businesses often achieve high levels of cooperation while engaging in exclusionary practices that we call parochialism. We investigate the contribution of within-group cultural affinity to the ability of parochial groups to cooperate in social dilemmas. We analyze parochial networks in which the losses incurred by not trading with outsiders are offset by an enhanced ability to enforce informal contracts by fostering trust among insiders. We show that there is a range of degrees of parochialism for which parochial networks can coexist with an anonymous market offering unrestricted trading opportunities.
\end{abstract}

(C) 2004 Elsevier B.V. All rights reserved.

PACS: C7; D2

Keywords: Parochialism; Exchange networks; Insider-outsider relationships

\section{Introduction}

Decentralized groups such as close knit residential neighborhoods and ethnically linked businesses often achieve high levels of cooperation while also engaging in exclusionary practices that we call parochialism. We investigate the contribution of within-group cultural affinity to the ability of parochial groups to cooperate in social dilemmas. Consistent with some recent experimental evidence, cooperation among members of culturally homogeneous groups does not come about because cultural affinity overrides individual self

\footnotetext{
* Corresponding author. Tel.: +1-413-586-7756; fax: +1-206-984-9873.

E-mail addresses: bowles@ santafe.edu (S. Bowles), hgintis@ comcast.net (H. Gintis).

URLs: http://www.santafe.edu/ bowles, http://www-unix.oit.umass.edu/ gintis.
} 
interest, thus leading members to behave altruistically towards others in their group. Rather, cultural affinity supports cooperation by altering the information structure of the interaction. We model the economics of ethnic networks, defined as sets of agents unified by similarity of one or more ascriptive characteristics engaged in non-anonymous interactions structured by high entry and exit costs, but lacking a centralized authority. ${ }^{1}$

Networks manage such common pool resources as fisheries, irrigation, and pasturage (Acheson, 1988; Wade, 1988; Ostrom, 1990), regulate work effort and risk sharing in producer cooperatives (Whyte, 1955; Homans, 1961; Lawler, 1973; Craig and Pencavel, 1992, 1995; Platteau and Seki, 2001), enforce non-collateralized credit contracts (Udry, 1993; Banerjee et al., 1994) promote neighborhood amenities in residential communities (Sampson et al., 1997), and privately enforce contracts among traders in securities (Baker, 1984) and diamond markets in the US (Bernstein, 1992), and food markets in Madagascar (Fafchamps and Minten, 2001).

Networks often do quite well economically, as the flourishing informal ethnic business linkages among new immigrants to the United States and the United Kingdom attest (Rauch, 1996; Granovetter, 1985; Kotkin, 1993). For instance, Cambodians run more than 80 per cent of California's doughnut shops, raising funds from friends, family, and ethnic credit associations (Kaufman, 1995). Similarly, Indians own more than a third of the motels in the United States, frequently raising initial capital through unsecured loans from extended family members (Woodyard, 1995).

Among the problem-solving capacities of networks are the powerful contractual enforcement mechanisms made possible by small-scale interactions, notably effective punishing of those who fail to keep promises, facilitated by close social ties, frequent and variegated interactions, and the availability of low cost information concerning one's trading partners. This problem-solving capacity allows networks to counteract the restricted gains from trade and foregone economies of scale due to small size and exclusionary practices. ${ }^{2}$ Members, of course, do not normally express their identification with networks in terms of their economic advantages. Rather, they typically invoke religious faith, ethnic purity, or personal loyalty. These sentiments often support exclusion or shunning of outsiders. We model these practices, which we term parochialism, in Section 2.

\footnotetext{
1 The theory of social exchange, initiated in sociology by Blau (1964) and Homans (1958), and in anthropology by Sahlins (1972) provide insights into the economics of networks. For contributions by economists, see Ben-Porath (1980), Hollander (1990), Iannaccone (1992), Kandori (1992), Wintrobe (1995), Greif (1994), Akerlof (1997), Pagano (1995), Bénabou (1996), Durlauf (1996), Kranton (1996), Taylor (1997), and Glaeser (2000).

2 The advantages of trade with those deemed "outsiders" is a common explanation of the permeability of network boundaries in small-scale societies (Adams, 1974) and of the extinction of very restrictive networks in favor of more inclusive entities (Gellner, 1985; Weber, 1976). A particularly well-documented example of this tension is Greif's account of how the competitive advantages stemming from the superior within-network contractual enforcement capabilities of the tight-knit 13th century community of Maghribi merchants was eventually offset by their reduced ability to engage in successful exchange with outsiders, resulting in their inability to compete with the more individualistic Genovese traders. Ben Porath (p. 14) develops similar reasoning concerning the economic capabilities of families and other face to face networks:
}

The transactional advantages of the family cannot compensate for the fact that within its confines the returns from impersonal exchange and the division of labor are not fully realizable. 
Networks arise in part because people choose to associate with others who are similar to themselves in some salient respect (Lazarsfeld and Merton, 1954; Thibaut and Kelly, 1959; Homans, 1961). Among the salient characteristics on which this choice operates are race and ethnic identification, and religion (Berscheid and Walster, 1969; Cohen, 1977; Kandel, 1978; Tajfel et al., 1971; Obot, 1988). Conversely, people often seek to avoid interactions with those who are different from themselves.

We seek to illuminate the following puzzle: why do parochial sentiments and practices, often identified with archaic social distinctions and intolerance of strangers, persist in modern market-based and liberal societies? Our response, briefly, will be that parochialism is not an anachronistic remnant of the past, perpetuated by inertia, but rather that networks based on parochialism solve economic problems that are resistant to market- or state-based solutions. Persistent parochialism is thus explained at least in part by the problem solving capacities of the network interactions that parochialism underpins.

When interactions among group members are characterized by material payoffs that take the form of prisoner's dilemma, public goods or other social dilemmas, parochialism may contribute to successful cooperation in three ways. First, members may feel more altruistic toward the ethnically similar members of their group than towards "outsiders". It is well known that if each member values the payoffs gained by other members sufficiently highly, mutual defect is no longer the dominant strategy equilibrium of these interactions, and mutual cooperation may be a stable Nash equilibrium. The "minimal group" experiments initiated by Tajfel et al. and his colleagues in the early 1970s are often interpreted in this way. Experimental subjects were assigned to groups on the basis of some trivial distinction (commonly their preference for paintings by Paul Klee over those of Wassily Kandinsky). In-group favoring behavior was quite pronounced in these experiments. Later prisoner's dilemma and common pool resource experiments found higher levels of cooperation when the players are members of the same minimal group than when they are not members of the same group (Kramer and Brewer, 1984). However, a series of recent experiments by Yamagishi (2003) and his associates show that experimental subjects' allocations favor in-group members not because of altruistic sentiments towards those who are similar to themselves, but because they expect reciprocation from in-groupers and not from out-groupers. ${ }^{3}$

A second way that cultural affinity could support cooperation is by enhancing the force of the forms of altruistic punishment that often sustain cooperation in experimental public goods games (Barr, 2001; Fehr and Gächter, 2002; Masclet et al., 2003). For example, it is plausible that the shame induced by criticism for selfish behavior from a fellow group member is greater when the critic shares the defector's beliefs about good behavior.

The third reason parochial groups may cooperate in interactions that would take the form of prisoner's dilemmas or public goods games in non-parochial settings, unlike the first two reasons, does not concern the members' preferences. Rather it is the effect of cultural affinity on the information structure of the interaction, allowing equilibrium strategies unavailable in the information environments of less parochial groups. Our model explores

\footnotetext{
3 Yamagishi's work does not show that within-group altruism does not exist. Rather, he shows that the minimal group experiments provide no evidence to this effect.
} 
this mechanism as a contributor to the success of parochial groups in addressing social dilemmas.

We do not suggest, of course, that the contribution of parochial sentiments and practices to economic performance of groups is the sole reason for their persistence. Ethnic, racial and other group identities arise and persist for a multitude of reasons, many of them far less benign than those studied here. Loury (2001) provides a compelling account of some of these reasons.

A successful model of parochialism in networks must satisfy four conditions. First, it should be a general equilibrium model in which the size of the anonymous market and the size and array of parochial networks are endogenously and simultaneously determined. We do this by constructing a model with explicit network-market interactions. Second, the range of ascriptive traits that can be the basis for a successful parochial network must be endogenously determined. We do this by developing the concept of ascriptive filters, each of which is associated in equilibrium with a network of a particular size and composition. Third, decision-making and information exchange in networks should be completely decentralized, thus distinguishing networks from teams with a centralized decision-making structure and groups all of whose members have access to a common pool of information. Finally, the exchanges that take place between group members must be one-shot, rather than being based on repeated dyadic interactions in which cooperation is ensured by superior gains of long-term interaction. This is not because most interactions within networks are one-shot as an empirical matter, but rather in order to illuminate what is distinctive about parochial networks. The major benefit of participating in a parochial network is the ability to gain information about a potential trade partner at low cost from other network members. Dyadic interactions that are sufficiently repeated operate effectively without such information. Our model meets all these requirements.

Our model is closest in spirit to McElreath et al. (2003), who show that ethnic markers are likely to be salient if they correlate with shared beliefs that facilitate cooperation. Like McElreath et al. (2003), ethnic affinity in our model does not support cooperation by means of positive sentiments among group members, but rather by removing barriers to exchange in the presence of incomplete contracts. Unlike their paper, however, we consider the distribution of a population between ethnically marked groups and an unmarked pool, and we model the reasons why shared ascriptive traits contribute to successful exchange, while also precluding some gains from trade. Our paper shares with the general equilibrium framework of interaction between a system of anonymous market exchange and another system of reciprocal relationships in small groups, as well as the notion that the comparative advantage of the anonymous market is in product diversity, while that of small groups is contract enforcement. However, Kranton's group relationships are dyadic interactions that discourage free-riding through repetition and the threat of withdrawal from the relationship, while in our model, all trades are one-shot events, free-riding being discouraged by a decentralized network information structure that establishes the reputation of group members. Like Greif, we consider the coexistence of alternative trading strategies. However, unlike Greif, we assume entirely decentralized information flows, the quality of which is affected by the degree of parochialism of the network. Cultural affinity of group members per se plays no role in either Greif's or Kranton's model. 
The mechanism for the success of networks explored in this paper is their ability to promote trust. ${ }^{4}$ We consider a large population of agents who, while economically identical, are distinguishable by markers indicating group membership. These agents take three types of actions. First, they locate in one of a variable number of networks or remain outside any network in what we will call the 'anonymous pool' of traders. Second, they choose strategies that govern their behavior with trading partners. Third, they update these strategies in light of their relative payoff compared to other available trading strategies. We explore the evolution and equilibrium frequency of behaviors within networks, the distribution of population between networks and the anonymous pool, and the size and number of networks, under the influence of parochial practices. We conclude with a series of implications of the model concerning the impact of the evolving information structure of modern economies on the likely future importance of parochial networks.

\section{Parochialism and heterogeneity in networks}

Individuals implement their desires to associate with others like themselves by engaging in what we term parochial practices. These practices take the form of refusal to trade with 'outsiders' that, ceteris paribus, lower the returns to members of parochial networks. McMillan and Woodruff (1999, p. 1285) study of trust among businesses in Vietnam suggests the salience of this tradeoff:

Trading relations in Vietnam's emerging private sector are shaped by two market frictions: the difficulty of locating trading partners and the absence of legal enforcement of contracts. Examining relational contracting, we find that a firm trusts its customer enough to offer credit when the customer finds it hard to locate an alternative supplier.... Customers identified through business networks receive more credit. These network effects are enduring, suggesting that networks are used to sanction defaulting customers.

Thus, in some cases, homogeneity may offer advantages offsetting the foregone gains from trade. Parochial communities such as the Pennsylvania Amish and the Canadian Hutterites have expanded their numbers and thrived economically. ${ }^{5}$ Among the Amish, for example, distinctive dress, dialect, and technology construct a "cultural moat" around the group and, acting as "armaments of defense, they draw boundary lines between church

\footnotetext{
${ }^{4}$ Our model develops insights provided by a number of contributions to the sociology of networks. Granovetter writes (pp. 490-491)

... social relations, rather than institutionalized arrangements or generalized morality are mainly responsible for the production of trust in economic life.

For additional ways in which networks solve coordination problems stemming from incomplete contracts, see Bowles and Gintis $(1998,2002)$.

5 See Wilson and Sober (1994) and Kraybill (1989). Hechter (1990) found that two indicators of group homogeneity, common ethnic background and uniform style of dress, were among the few robust predictors of survival of utopian communes established in the late 18th and early 19th century in the United States. He interprets this finding as in part reflecting variable information costs. See also Longhofer (1996) for a model of the relationship between cultural affinity and monitoring costs.
} 
and world [to] announce Amish identity to insider and outsider alike" (Kraybill 50,68). Yet the boundaries erected around Amish culture have not prevented economic success and population growth. Further, the record of successful ethnic business affiliations suggests that parochialism may not only foreclose opportunities, but also contribute to the success of networks.

We model parochialism as an ascriptive on given traits of those with whom one might interact, a particular form of parochialism excluding those with 'objectionable' traits. ${ }^{6}$ Individuals who do not exclude those with objectionable traits are themselves objectionable, even if their traits per se are not objectionable. ${ }^{7}$ Thus any ascriptive different from one's own is assumed to be objectionable, so networks will made up of individuals with the same type of parochialism.

It might be thought that this assumption presents an incentive problem for members whose traits conform to the filter but who stand to gain by secretly transacting with agents whose traits do not conform. This is not the case. To see this, suppose network members trade only among themselves. Then, an agent who considered trading with a non-member would know that he could obtain no information concerning the non-member's behavior. The non-member would have no incentive to act cooperatively; there would be no reputation value of doing so because he does not trade with other network members. Hence such a trade would have the payoffs of an anonymous market transaction, but if such a trade were beneficial, the member would be better off in leaving the network entirely. As a result, however different they are in other respects (for example, pursuing different strategies in economic interactions or differing in a trait not covered by the filter), they will agree on the common traits for which their ascriptive filter selects. ${ }^{8}$

In principle, any individual trait, such as liking the color blue or eating lots of fish, could be the basis for eligibility for membership in a parochial network. Some characteristics, including such ascriptive traits as race, ethnicity, and native language, however, are much more conducive to network formation. First, such ascriptive characteristics are difficult to fake or acquire, thus solving the problem of limiting immigration into the network when it is successful. Second, individuals with shared ascriptive traits are likely to have shared values, means of communication, beliefs, and tastes. Hence, two members of an ethnic network are more likely to agree on and communicate what counts as acceptable trading behavior of a third party than are two randomly chosen members of the population who happen to agree on a trivial and easily changed characteristic.

\footnotetext{
${ }^{6}$ Iannaccone analyzes a more active form of parochialism, in which membership in a network subject to participatory crowding is restricted to those who are willing to accept "stigma, self-sacrifice, and bizarre behavioral restrictions".

${ }^{7}$ Lazarsfeld and Merton term this second order exclusiveness "value homophily" and present evidence for it with respect to racial attitudes: white 'racial liberals' prefer not to associate with white 'racial illiberals' and conversely. Sugden (1986) showed this to be a coherent basis for group formation, using the concept of being "in good standing" in the group. An individual who lacks one or more of the group's core characteristics is not in good standing. An individual who associates with an individual who is not in good standing is himself not in good standing. Otherwise, an individual is in good standing. A network consists of all individuals who are in good standing with respect to its core characteristics.

${ }^{8}$ The assumption that ethnic network members share a common filter is justified empirically, but relaxing it would not qualitatively alter our analysis, while complicating it considerably. To the extent that network members have disparate filters, information exchange will be more costly and less efficient.
} 
Suppose in pairwise strategic interactions, agents can condition their actions on whether the other player is an 'insider' or an 'outsider'. Each individual has a certain set of traits (ethnicity, language, physical attributes, cultural or demographic characteristics, and the like), which we take to be fixed. We label these traits $j=1, \ldots, n$, each individual being characterized by a trait profile $a=a_{1}, \ldots, a_{n}$, where each $a_{j}=1$ or $a_{j}=0$ according as to whether the individual does or does not possess trait $j$. Let $A$ be the set of all possible trait profiles. An individual with traits $a \in A$ may have a 'ascriptive filter', defined as a vector $b \in A$ such that $b \leq a$, in the sense that the individual with traits $a$ also has all the traits indicated by $b$.

Let us define an individual as $b$-parochial if he has all the $b$-traits, and he trades only with other $b$-parochial individuals. We also refer to $b$-parochial agents as insiders (the trait vector $b$ being assumed), and we refer to a non-insider as an outsider. A outsider is therefore an individual who lacks one or more of the $b$-traits, who trades with someone who lacks one or more of these traits, or who trades with someone who trades with someone who lacks one or more of these traits, and so on. In effect, $b$-parochial agents choose a subset of the traits they possesses (the unit-entries in $b$ that are also unit-entries in $a$ ) and consider as insiders exactly those agents who have these traits and are 'like-minded' in the sense that they have the same criteria for distinguishing between insiders and outsiders. We assume throughout that the property of being $b$-parochial is common knowledge.

This formalization reflects our view that the immense variety of noticeable individual differences and similarities is the raw material on which parochialism works. A particular $b$-parochialism makes some subset of these differences behaviorally salient while ignoring others. For instance, suppose the array of traits are ('female', 'French-speaking'). An agent with characteristics $a=11$ is a female Francophone. Such an individual could be $b$-parochial for $b=11$ (insiders are like-minded female Francophones), $b=01$ (insiders are like-minded Francophones), $b=10$ (insiders are like-minded females), or $b=00$ (insiders are like-minded i.e., they treat all others as insiders).

It is clear from this example that individuals may differ in the extent of their parochialism. As we will see below, these differences will affect both the size and heterogeneity of networks, which in turn will influence the gains from within-network trading, but first we need to formalize the degree of parochialism of a network and the expected communication difficulty within a network.

Suppose, for example, there are three salient binary traits, language, nationality, and "race". We define trait profiles so that a person with the trait profile $a=111$ is Frenchspeaking, European, and "white", while a 000 is non-French-speaking, non-European, and non-white. A 010 person is a non-French-speaking, European non-white, and so on, covering all eight possible trait types generated by these categories. The degree of parochialism, $\rho$, is a measure of the stringency of an individual's filter, as indicated by the minimum number of ways another must resemble the individual in order to be considered an insider. Thus $\rho=0$ indicates the total absence of parochialism, while $\rho=3$ indicates complete parochialism (for the three-trait case), meaning that such individuals will associate only with those identical to them in all three traits. Because networks will be homogeneous with respect to the degree of parochialism, we can speak of $\rho$ as a network trait.

We cannot in general compare the degree of parochialism between arbitrary filters. We can, however, determine which of two filters that differ only in one entry is more parochial. 
For instance, we cannot say that excluding Jews or excluding blacks is more parochial, but we can say that excluding Jews and women is more parochial than simply excluding Jews. By extension, we can compare two filters if their differences can be expressed by a series of such comparisons. In other words, ascriptive filters partially order the set $\mathcal{P}$ of parochial networks. ${ }^{9}$ Given networks $\mathcal{N} \in \mathcal{P}$ consisting of $b(\mathcal{N})$-parochial agents and $\mathcal{M} \in \mathcal{P}$ with $b(\mathcal{M})$-parochial agents, we say $\mathcal{M}$ is more parochial than $\mathcal{N}$ if $b(\mathcal{M})>b(\mathcal{N})$, so every member of $\mathcal{M}$ would be admitted to $\mathcal{N}$.

To explore the impact of the degree of parochialism on the ability of network members to cooperate effectively, we assume that communication difficulty rises with the number of trait differences. Let $\mu_{i j}$ represent the communication difficulty between individuals of trait types $i$ and $j$, defined as the number of traits on which they differ. In the three trait case, for instance $\mu_{i j}$ can take on the values $0,1,2$, and 3 .

Now consider the communication difficulty arising among randomly paired individuals in networks with a given ascriptive filter. Suppose there are $m$ types of agents in the network, where the frequency of type $i$ is $p_{i} \geq 0, i=1, \ldots, m$. Assuming random pairing of agents, the expected communication difficulty is then given by

$$
\mu=\sum_{i, j=1}^{m} p_{i} p_{j} \mu_{i j} .
$$

For instance, continuing our previous example, assume that each of the eight trait types is equally common in the larger population from which the networks are drawn and that the relative frequency of types who are admitted to a network is equal to their relative frequency in the larger population. Then if $\rho=3$, there are no communication difficulties, as all members of the network have the same three traits, so $\mu=0$. If $\rho=2$, by contrast, the network will be composed of equal numbers of two types similar with respect to two traits and different with respect to the other trait. Thus a random pairing will yield pairs with a one-trait difference approximately half the time, yielding an expected communication difficulty $\mu=1 / 2$. By similar reasoning $\rho=1$ yields $\mu=1$ and $\rho=0$ gives $\mu=3 / 2$. This reasoning is readily generalized to larger numbers of traits and trait groups of unequal size.

We can show that $\mu$ is decreasing in $\rho$ on any totally ordered subset of the set $\mathcal{P}$ of parochial networks. Consider a network $\mathcal{N} \in \mathcal{P}$, and suppose there are $m$ types in $\mathcal{N}$, type $i$ occurring with frequency $p_{i}$. In addition, consider the more parochial network $\mathcal{M}$ gotten by replacing members of $\mathcal{N}$ who lack a certain previously ignored trait, say trait 1 , with agents who possess this trait and have otherwise identical trait profiles as the agents they replace. Then, provided that the fraction of agents in $\mathcal{N}$ with trait 1 lies strictly between zero and one, members of the newly constituted, more homogeneous, network $\mathcal{M}$ will enjoy strictly less communication difficulty. To see this, note that before the change an agent had a positive change of meeting an agent with a different value for trait 1 , and now has a zero chance. Moreover, no other meeting probability has changed, so communications costs must fall for all agents. We have the following theorem.

\footnotetext{
${ }^{9}$ Formally, a partial ordering < on a set $S$ is a transitive binary relation on $S$, and a total ordering on $S$ is a partial ordering such that, for any two elements $A, B \in S$, either $A=B, A<B$, or $B<A$.
} 
Theorem 1 (Parochialism and communication difficulty). Increasing parochialism in any totally ordered subset of the set of parochial networks $\mathcal{P}$ reduces communication difficulty $\mu$.

\section{The costs and benefits of networks}

In this section, we analyze the effect of the degree of parochialism on the information and trading opportunities available to members of a single network, taking as given the composition of and payoffs to members in other networks and the anonymous pool of traders. Theorem 1 shows that level of expected communication difficulty $\mu(\rho)$ is decreasing in $\rho$ on any totally ordered subset $\mathcal{P}^{\mathrm{o}} \subset \mathcal{P}$. We shall assume network size $x(\rho)$ is decreasing in $\rho$ on $\mathcal{P}^{0}$, for the obvious reason that increased parochialism reduces the pool of potential migrants to the network. We will later examine the manner in which the size $x(\rho)$ of a single network depends on the composition of other networks and the anonymous pool.

Suppose members of any network $\mathcal{N} \in \mathcal{P}^{0}$ are either trustworthy or untrustworthy (we model trustworthiness in Section 4). We will call the trustworthy members cooperators and the untrustworthy members defectors. We will show that for agents trading within a network the quality of the signal $p(\rho)$ is an increasing function of $\rho$ on $\mathcal{P}^{0}$, and the probability $q(\rho)$ of meeting a partner for mutually beneficial trade is a decreasing function of $\rho$ on $\mathcal{P}^{o}$. Signal quality $p(\rho)$ is increasing in $\rho$ on $\mathcal{P}^{0}$ for two reasons. First, more parochial networks are smaller, and smaller networks possess more information concerning each individual. Second, more parochial networks have lower communication costs, leading to a higher likelihood of correctly ascertaining the trustworthiness of potential trading partners. Similarly, $q(\rho)$ is decreasing in $\rho$ on $\mathcal{P}^{0}$ because more parochial networks have fewer members, and hence each member faces a lower probability of meeting a potentially mutually beneficial trading partner. Moreover, a more homogeneous set of agents is less likely to enjoy complementary patterns of excess supply and demand.

For any totally ordered set of networks $\mathcal{P}^{0} \subset \mathcal{P}$, we define a network information structure $I\left(x(\rho), \kappa, p_{\mathrm{o}}(\rho)\right)$ with the following properties. Each member of a network of $x(\rho)$ individuals knows the type (cooperator/defector) of $\kappa$ other members. An individual who seeks to known the type of a specific member $j$ of the network receives informant messages randomly from members of the network, until a message arrives from an informant who knows $j$ 's type. The informant's report of $j$ 's type is correctly communicated with probability $\tau$, which varies inversely with $\mu(\rho){ }^{10}$

We can then express the probability that the individual receives the correct information, $p(\rho)$ as follows. Let $q$ be the probability of receiving correct information if the agent does not know his partner. Then

\footnotetext{
${ }^{10}$ Note that an incorrect communication can occur either because the informant errs, or because the informant lies. There is no gain to lying in our model, so it is permissible to treat willful misinformation as a stochastic event that does not vary systematically with the variables and parameters of our model.
} 


$$
\begin{aligned}
& p=\frac{\kappa}{x}+\left(1-\frac{\kappa}{x}\right) q, \\
& q=\frac{\kappa}{x} \tau+\left(1-\frac{\kappa}{x}\right) q .
\end{aligned}
$$

Eqs. (1) and (2) have the following interpretation. With probability $\kappa / x$ person $j$ is known to the individual, but with probability $(1-\kappa / x)$ the individual, not knowing $j$ personally, must consult an informant. The informant will know $j$ with probability $k / x$ and will communicate this successfully to the individual with probability $\tau$. However with probability $(1-\kappa / x)$ the informant will not know $j$, and the individual must seek another informant, yielding the recursion expressed above.

Eqs. (1) and (2) may be solved as

$$
p(\rho)=\frac{\kappa}{x(\rho)}+\left(1-\frac{\kappa}{x(\rho)}\right) \tau(\rho) .
$$

Clearly $p(\rho)$ is increasing in $\rho$, since $x(\rho)$ is decreasing and $\tau(\rho)$ is increasing. Therefore we have the following theorem.

Theorem 2 (Parochialism and signal quality). Consider a totally ordered subset $\mathcal{P}^{0} \subset \mathcal{P}$ of parochial networks, and let $I(x(\rho), \kappa, \tau(\rho))$ be the information structure of a network in $\mathcal{P}^{o}$. Then the average signal quality $p(\rho)$ on $\mathcal{N}$ is an increasing function of the level of parochialism.

We model this as a problem of finding partners with whom to exchange goods or services, but an equivalent formulation would model the problem of finding advantageous matchings for some joint production activity with skill complementarities between demographic groups giving an advantage to more heterogeneous group. To specify the shape of $q(\rho)$ on $\mathcal{P}^{0} \subset \mathcal{P}$, suppose agents produce goods for trade in the morning and take them to market for trade in the afternoon. Goods are perishable and cannot be stored. Suppose there are $x(\rho)$ agents in the network and there are goods $1, \ldots, k$, corresponding to which there are 'marketplaces' that have exogenously given relative sizes $f_{1}, \ldots, f_{k}\left(\sum_{i} f_{i}=1\right)$. Marketplace $i$ thus has absolute size $x_{i}=f_{i} x(\rho)$ for $i=1, \ldots, k$. The members who are to compose this $x_{i}$ are assigned randomly at the start of the trading period. Each agent decides to be a buyer or a seller that period. Buyers and sellers in the same marketplace are randomly paired, and if the number of buyers and sellers differ, a random selection of agents will make no trade at all, and as a result trades on the anonymous market, receiving a payoff normalized to zero. Suppose the distribution of individual capacities and preferences differ among groups, so a network composed of many groups will have a greater variance of both preferences and production possibilities than a homogeneous group. To capture the effect of heterogeneity on the probability of trade, we assume that agents of the same type are more likely to be located on the same side of the market. Thus the expected fraction $\psi_{i}(\rho)$ of agents on the demand side of marketplace $i$ will be distant from 1/2 when networks are very homogeneous and close to $1 / 2$ when networks are heterogeneous.

The more parochial a network is, the less likely will agents be able to make a trade, for two reasons. First, the more parochial networks will be more homogeneous, so bunching many agents on one side of the market will happen frequently. Second, even if the expected 
number of buyers and sellers were equal in every market, the smaller each market is, the more likely mismatches will be on any particular market day. Theorem 3, reflecting this logic, is proved in Appendix A.

Theorem 3 (Gains to network heterogeneity). Let $q(\rho)$ be the probability of making a trade when network parochialism is $\rho$. Then $q(\rho)$ is decreasing in $\rho$.

\section{Trust in networks}

To model the population of traders, consider a game $G$ where many agents are randomly paired to play a one-shot prisoner's dilemma in which each receives $c$ if they both defect, each receives $b$ if they both cooperate, and a defector receives $a$ when playing against a cooperator, who receives $d$. The assumptions of the prisoner's dilemma then require $a>$ $b>c>d$ and $2 b>a+d$ (the latter inequality ensuring that mutual cooperation yields higher average payoffs than defect/cooperate pairs). The coordination failure underpinning the prisoner's dilemma structure of this interaction arises because some aspects of the goods or services being exchanged are not subject to costlessly enforceable contracts. The defect strategy, for example could represent supplying shoddy goods where product quality is not subject to contract.

We assume that any agent can trade in the anonymous market, the payoff to which we normalize to zero. As agents in this market are unknown to one another, their interactions are effectively non-repeated, precluding the kinds of informal contractual enforcement that may be possible for interactions within networks. It is reasonable to suppose that the kinds of goods and services traded in the anonymous market will tend to be those for which relatively complete and easily enforceable contracts can be written. Networks, by contrast, may specialize in the exchange of more difficult to contract goods and services.

An agent in a network can refuse to trade with his current partner, in which case we assume he trades on the anonymous market instead. If an agent does trade within the network, the payoffs to mutual cooperation exceed the payoffs available in the anonymous market $(b>0)$ but at the same time the payoffs to mutual defection are inferior to the payoffs of trades in the anonymous market $(c<0)$. This assumption is based on the notion that with incomplete contracting, trading agents expose themselves to a greater level of harm than would be the case with complete contracting. Thus operating within a network is disadvantageous compared with operating in the anonymous market, unless the level of cooperation within the network is sufficiently high.

We assume that each agent precommits to following one of three available 'norms'. The first, which we call Defect, is to defect unconditionally against all partners. The second, which we call Trust, is to cooperate unconditionally with all partners. The third, which we call Inspect, is to monitor an imperfect signal based on information provided by other members of the network indicating whether or not one's current partner defects against cooperators. We assume the signal correctly identifies a Defector with probability $p$ and correctly identifies a non-Defector with the same probability $p$. The Inspector then refuses to trade with a partner who is signaled as a Defector and otherwise plays the cooperate strategy. Thus when either partner to a within-network exchange refuses to trade, each 


\begin{tabular}{|c|c|c|c|}
\hline & Inspect & Trust & Defect \\
\hline Inspect & $b p^{2}, b p^{2}$ & $b p, b p$ & $d(1-p), a(1-p)$ \\
\hline Trust & $b p, b p$ & $b, b$ & $d, a$ \\
\hline Defect & $a(1-p), d(1-p)$ & $a, d$ & $c, c$ \\
\hline
\end{tabular}

Fig. 1. The Inspect-Trust-Defect game.

receives payoff 0 which, according to the reasoning of the previous paragraph, is better than the mutual defect payoff $c<0 .{ }^{11} \mathrm{We}$ assume that the signal is costlessly observed. Assuming a (not excessively large) positive cost of inspecting changes our results in an intuitively expected way, so we abstract from such costs in the interests of simplicity. The payoff matrix for a pair of agents has the normal form shown in Fig. 1. We write $G(p)$ for the game with signal accuracy $p$.

Let $\alpha_{t}, \beta_{t}$, and $\delta_{t}$ be the fraction of the population playing Inspect, Trust, and Defect at time $t$, respectively. We assume these are continuous variables. Let $\pi_{\mathrm{I}}^{t}, \pi_{\mathrm{T}}^{t}$, and $\pi_{\mathrm{D}}^{t}$ be the payoffs to the strategies Inspect, Trust, and Defect at time $t$, respectively, against the mixed strategy given by $\left(\alpha_{t}, \beta_{t}, \delta_{t}\right)$. We find that

$$
\begin{aligned}
& \pi_{\mathrm{I}}^{t}=b p\left(p \alpha_{t}+\beta_{t}\right)+d(1-p) \delta_{t}, \\
& \pi_{\mathrm{T}}^{t}=b\left(p \alpha_{t}+\beta_{t}\right)+d \delta_{t}, \\
& \pi_{\mathrm{D}}^{t}=a\left(\alpha_{t}(1-p)+\beta_{t}\right)+c \delta_{t}, \\
& \bar{\pi}^{t}=\alpha_{t} \pi_{\mathrm{I}}^{t}+\beta_{t} \pi_{\mathrm{T}}^{t}+\delta_{t} \pi_{\mathrm{D}}^{t},
\end{aligned}
$$

where $\bar{\pi}^{t}$ is the average payoff in the game. Equating the payoffs to the three pure strategies, we find that the Nash equilibrium frequencies $\left(\alpha^{*}, \beta^{*}, \delta^{*}\right)$ satisfy

$$
\begin{aligned}
& \alpha^{*}=\frac{-a d p+b(d(2 p-1)+c(1-p))}{D}, \\
& \beta^{*}=\frac{p(a d(1-p)-b(d(2 p-1)+c(1-p)))}{D}, \\
& \delta^{*}=\frac{a b(1-p)(2 p-1)}{D},
\end{aligned}
$$

where

$$
D=a\left(b(1-p)(2 p-1)-d p^{2}\right)+b(1-p)(d(2 p-1)+c(1-p)) .
$$

We have the following theorem.

Theorem 4 (A trust equilibrium). There is a $p_{*}<1$ such that for $p_{*}<p<1, G(p)$ has a unique Nash equilibrium $\left(\alpha^{*}(p), \beta^{*}(p), \delta^{*}(p)\right)$. In this equilibrium all three types of players

\footnotetext{
11 It is easy to show that other actions available to an Inspector who receives a signal indicating a defecting partner involve either mimicking the behavior of Trusters or Defectors or else are strictly dominated by playing as indicated above. We thus lose nothing by ignoring such alternatives.
} 
occur as strictly positive fractions of the population. The payoff $\pi^{*}(p)$ in this equilibrium is positive and an increasing function of $p$, the fraction of Defectors $\delta^{*}(p)$ is a decreasing function of $p$, and the fraction of Trusters is an increasing function of $p$.

To prove the theorem, define

$$
p_{*}=\max \left[1-\frac{c}{d}, \frac{a}{2 b}\left(\sqrt{\frac{4 b}{a}+1}-1\right)\right] .
$$

Note that $p_{*} \in(0.618,1)$, since $d<c<0$ and $a>b>0$. Then it is easy to show that

$$
d(1-p)>c \quad \text { and } \quad b p^{2}>a(1-p)
$$

for all $p$ such that $p_{*}<p<1$. The inequalities in (11) imply that Inspect is a best response to Defect, so mutual defect cannot be an equilibrium, and that Defect is not a best response to Inspect, so a Defect-Inspect equilibrium is precluded. A routine check then indicates that there are no Nash equilibria involving fewer than all three strategies. ${ }^{12}$ Hence by Nash's existence theorem, there is an equilibrium of $G(p)$ involving all three strategies. This proves that $p_{*}$ has the asserted property. Eqs. (8)-(9) imply

$$
\pi^{*}=\frac{-a b d(2 p-1)^{2}}{D}
$$

so $\pi^{*} / \delta^{*}=-d(2 p-1) /(1-p)>0$, showing that payoffs are positive. A tedious calculation verifies that

$$
\frac{\mathrm{d} \pi^{*}}{\mathrm{~d} p}=\delta^{* 2}(-d) \frac{b(d(2 p-1)+2 c(1-p))+a(b(2 p-1)-2 d p)}{a b(1-p)^{2}(2 p-1)} .
$$

The denominator in the fraction is positive and the numerator can be written as

$$
2(b(a-c)-d(a-b))\left(p-\frac{1}{2}\right)+b c-d a,
$$

which is clearly positive. To prove the final assertion, we calculate

$$
\frac{\mathrm{d} \delta^{*}}{\mathrm{~d} p}=\frac{\delta^{* 2} a b(2 p-1)^{2}(1-p)^{2}}{b c(1-p)^{2}+a d p(3 p-2)} .
$$

The denominator in this expression is less than $b c(2 p-1)^{2}<0$, from which the assertion follows.

The intuition behind Theorem 4 is simple. Consider the simplex

$$
T=\{(\alpha, \beta) \mid \alpha, \beta, \alpha+\beta \in[0,1]\} .
$$

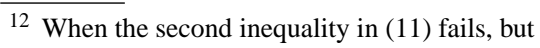

$$
p<\frac{(a / b)+(1-(c / d))}{1+(a / b)+(1-(c / d))}
$$

there also is no Nash equilibrium involving fewer than three pure strategies. We will ignore this alternative, for ease of exposition. 


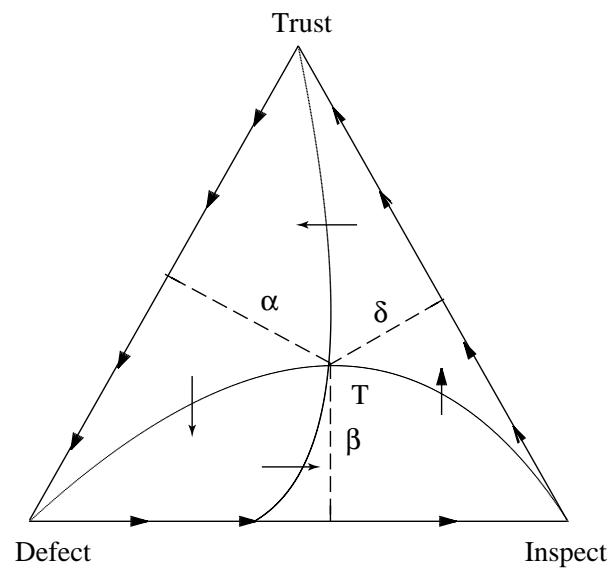

Fig. 2. A simplex phase diagram for $G(p)$ when $p_{*}<p<1$. The frequency of Inspect, Trust, and Defect are $\alpha$, $\beta$ and $\delta$, respectively. The trust equilibrium is at T. Note that there are no equilibria along the two-dimensional boundary of the simplex, since each pure strategy can be invaded by another. The solid interior lines divide the simplex into regions with differing dynamics. The line from $\mathrm{T}$ to the all Trust vertex is the locus of points such that $\mathrm{d} \beta / \mathrm{d} t=0$ while $\mathrm{d} \delta / \mathrm{d} t>0$ and $\mathrm{d} \alpha / \mathrm{d} t<0$, for example, so a group with a composition at a point on this locus point will evolve in the direction given by the arrow.

By Nash's existence theorem there is an equilibrium within T. However Trust is strictly dominated by Defect, and Inspect is strictly dominated by Trust (since Inspectors refuse some profitable trades, while Trusters do not). When the two inequalities (12) hold, Defect is also strictly dominated by Inspect. Therefore all Nash equilibria must be confined to the interior of T. However, it is easy to check that there is only one possible candidate, which thus exists and is unique. A phase diagram for the model is presented in Fig. $2 .{ }^{13}$

The replicator equations are then given by

$$
\begin{aligned}
\frac{\mathrm{d} \alpha_{t}}{\mathrm{~d} t} & =\alpha_{t}\left(\pi_{\mathrm{I}}^{t}-\bar{\pi}^{t}\right), \\
\frac{\mathrm{d} \beta_{t}}{\mathrm{~d} t} & =\beta_{t}\left(\pi_{\mathrm{T}}^{t}-\bar{\pi}^{t}\right),
\end{aligned}
$$

reflecting our assumption that norms are implicated in the response to relative payoffs.

We then have the following theorem.

Theorem 5 (Stability of the trust equilibrium). For $p>p_{*}$, the unique equilibrium $P=$ $\left(\alpha^{*}, \beta^{*}, \delta^{*}\right)$ of $G(p)$ either is stable or has paths starting sufficiently near $P$ that converge

\footnotetext{
13 We must also check on the dynamic properties of the interior Nash equilibrium. There is no guarantee that this equilibrium is evolutionarily stable. Indeed, the reader can check that for $a=2, b=1, c=-1$ and $d=-2$, the equilibrium is not evolutionarily stable for $p \geq 0.78$, while if we change $a$ to $a=3$, it is evolutionarily stable. However, evolutionary stability is a sufficient, though by no means necessary, condition for dynamic stability (Gintis, 2000, Chapter 10). Therefore, we must inspect a plausible dynamic, which we take to be the replicator dynamic (Taylor and Jonker, 1978).
} 
to a periodic orbit of the replicator dynamic. In the latter case, the time averages of the payoffs along the periodic orbit for the three strategy types are all equal to $\pi^{*}(p)$. Thus in either the stable or limit cycle case, the long-run expected payoff to an agent is $\pi^{*}(p)$, which is an increasing function of the signal quality $p$.

The first assertion follows directly from the Poincaré-Bendixson Theorem (Perko, 1991, p. 227), and the second from an ergodic theorem-Theorem 7.6.4 in Hofbauer and Sigmund (1998, p. 79). By virtue of this theorem, we will therefore refer to either the stable or limit cycle case as a stable equilibrium of $G(p)$.

It is easy to check that when $p<p_{*}$, there are only All Defect, or Defect/Inspect equilibria, both of which yield negative expected payoff. The first is stable and the second unstable in the replicator dynamic. We assume the network disbands in such cases, so we take $\pi^{*}(p)=0$ for $p<p_{*}$.

\section{The limits of sustainable parochialism}

The characterization of the equilibrium given by Theorems 4 and 5 allow us now to consider the effects of varying the degree of parochialism on the payoff to network members.

Consider the game $G^{\prime}(\rho)$, where $\rho$ is the degree of network parochialism, differing from $G$ in two ways. First, the payoff to the prisoner's dilemma stage game is the payoff in $G$ multiplied by the decreasing function $q(\rho)$ (see Section 3) minus a fixed cost $\bar{c}>0$ of seeking a within-network transaction. Second, we assume the probability $p$ of correctly identifying the type of a potential trade partner is an increasing function $p=p(\rho)$ within any totally ordered set $\mathcal{P}^{0} \subset \mathcal{P}$ of networks, as per Theorem 2 . We call the game $G^{\prime}(\rho)$ the variable parochialism network game.

We assume that there is a degree of parochialism $\rho_{\min }$ on $\mathcal{P}^{0}$, such that $p(\rho)>p_{*}$ for $\rho>p_{\min }$. Thus there is a stable interior equilibrium for $G^{\prime}(\rho)$ for $\rho>p_{\min }$. The equilibrium payoff in $G^{\prime}(\rho)$, for $\rho>p_{\min }$ is then

$$
\bar{\pi}(\rho)=q(\rho) \pi^{*}(p(\rho))-\bar{c}
$$

and the equilibrium frequencies of Inspectors and Trusters can be written as $\alpha^{*}(p(\rho))$ and $\beta^{*}(p(\rho))$ on $\mathcal{P}^{0}$, respectively.

Theorem 6 says that if the number of agents of a particular type are either too small or too large, this type cannot sustain a network equilibrium. This reflects the common observation that networks generally consist of minorities, but when insufficiently numerous, such minorities can do no better than operate within the anonymous pool because trading opportunities become too rare to offset the transactions cost $\bar{c}$ of seeking a within-network trade. Similarly, when the network becomes too large, signal quality becomes too low to support a trust equilibrium. We have the following theorem.

Theorem 6 (Equilibrium network size). Let $\mathcal{P}^{0} \subset \mathcal{P}$ be a totally ordered set of networks, so that each network $\mathcal{N} \in \mathcal{P}^{0}$ is characterized by a particular parochialism level $\rho$. For sufficiently small transactions cost $\bar{c}$, there is a non-empty interval $\left(x_{\min }, x_{\max }\right)$ such that a 


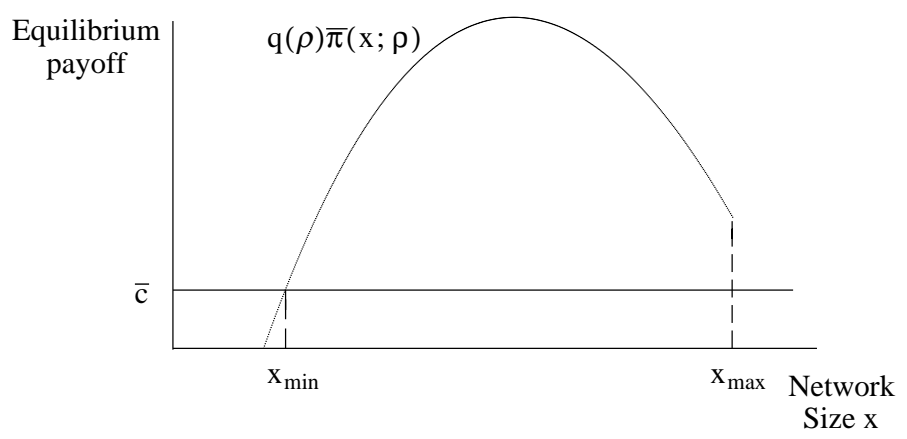

Fig. 3. Payoffs in a trust equilibrium and network size for a network with a given level of parochialism. Note that $x_{\max }$ is given by (17), and $x_{\min }$ is determined implicitly by the equation $q(\rho) \bar{\pi}(\rho)=\bar{c}$.

trust equilibrium that is stable in the replicator dynamic exists if and only if $x_{\min }<x(\rho)<$ $x_{\max }$.

To prove the theorem, we equate $p(\rho)$ in (3) with $p_{*}$ in (11) and solve for $x$, which gives

$$
x_{\max }=\min \left[\frac{d \kappa(1-\tau)}{c+d(1-\tau)}, \frac{2 b \kappa(1-\tau)}{\sqrt{a^{2}+4 a b}-(a+2 b \tau)}\right]
$$

as the maximum feasible network size for the stage game. Now (16) shows that for sufficiently small $\bar{c}>0$, equilibrium profits are strictly positive in the variable parochialism game as well. To determine $x_{\min }$, we first find $\rho_{\min }$, the level of parochialism such that expected payoffs in a trust equilibrium are zero (by setting (16) equal to zero and solving for $\rho)$ and then letting $x_{\min }=x\left(\rho_{\max }\right)$. We illustrate this situation in Fig. 3.

Note that it will generally be the case that $x_{\min }$ and $x_{\max }$ differ across totally ordered subsets of ascriptive filters. The reason is that one subset may implement large size with little heterogeneity (thus allowing a larger $x_{\max }$ as the information costs of larger size would be partially offset by lesser communication difficulty) while another subset may implement a high level of heterogeneity even for relatively small size. Similarly, if equilibrium payoffs differ across networks (because of different parameters $a, b, c, d, \tau, k$ and $\bar{c}$ ), $x_{\min }$ will also vary.

\section{Parochialism and the anonymous market}

In our model, individuals not in networks make up the anonymous pool of traders, unconditionally defecting and receiving a payoff normalized to zero. We now study a population-level equilibrium in which agents may migrate among one or more networks and the anonymous pool, and they do so when movement would increase their expected payoffs. For simplicity, we assume the cost of movement is zero and that networks accept all immigrants who satisfy the network's ascriptive filter. We will identify the conditions under which parochial networks may survive under these conditions. 
It is easy to see that if ascriptive filters are very coarse such that, given some population composition, a limited range of sizes of groups is possible, it may be that no feasible network falls within the $x_{\min }$ and $x_{\max }$ identified in Theorem 6 . Our first condition for the presence of parochial network is that at least one filter be sufficiently fine in the following sense. Since there are $n$ traits, there are $2^{n}$ possible trait profiles $a \in A$. Let $f(a)$ be the frequency of profile $a \in A$ in the population, and let $F(a)$ be the fraction of the population that satisfies ascriptive filter $a \in A$. We thus have

$$
F(a)=\sum_{b \geq a} f(b) .
$$

For simplicity, we suppose the cost to an agent of moving from the anonymous pool to a network that will accept him is zero, and networks accept all immigrants who satisfy the network's ascriptive filter. On any totally ordered subset $\mathcal{P}^{0} \subset \mathcal{P}$, we can associate the degree of parochialism $\rho$ with a particular ascriptive filter $a(\rho) \in A$, in which case we have $x(\rho)=F(a(\rho))$. Moreover, if $\mathcal{P}^{0}$ is a maximal totally ordered subset, $F(a(0))=1$ and $F(a(n))=0 .{ }^{14}$ We call a maximal totally ordered set $\mathcal{P}^{0} \subset \mathcal{P} \epsilon$-fine if, for every filter $a_{N}$ of a network $\mathcal{N} \in \mathcal{P}^{o}$, there is a network $\mathcal{M} \in \mathcal{P}^{o}$ with associated filter $a_{M}$ such that $\left|F\left(a_{N}\right)-F\left(a_{M}\right)\right|<\epsilon$. Let $X$ be the size of the population. We then have the following theorem.

Theorem 7. Suppose there is an $\epsilon$-fine maximal totally ordered subset $\mathcal{P}^{0} \subset \mathcal{P}$, where $\delta=\left(x_{\max }-x_{\min }\right) / X$ for $x_{\max }$ and $x_{\min }$ given by Theorem 6 . Then there exists an ascriptive filter a and a network $\mathcal{N}$ using ascriptive filter a that is a stable equilibrium of the replicator dynamic.

Note that in equilibrium, all members of the population who satisfy the ascriptive filter $a$ migrate from the anonymous pool to the network $\mathcal{N}$.

Under the conditions given in Theorem 7, there must exist a filter and a level of parochialism implementing a group size within the given range, which by Theorem 6 supports a stable equilibrium with an average payoff $\bar{\pi}>0$. If there are no other networks, the network in question will attract all population members conforming to the filter.

Suppose now that agents can move not only to networks costlessly, but can also move among networks costlessly. We define a population-level equilibrium as a set of networks $\mathcal{N}_{1}, \ldots, \mathcal{N}_{k}$ such that (a) each network is a stable equilibrium of the replicator dynamic; (b) no individual can gain by moving from the anonymous pool to a network; (c) no individual can gain by moving from one network to another network; and (d) there is no ascriptive filter a such that a network based on a could draw individuals from either one of the existing networks or from the anonymous pool. We have the following theorem.

Theorem 8. Suppose the conditions of Theorem 7 are satisfied. Then there is a populationlevel equilibrium with at least one network.

\footnotetext{
14 This assumes that no agent has all $n$ traits, which will be the case, for instance, if the traits include national origin.
} 
To prove this theorem, let $\mathcal{N}_{1}$ be the viable network with highest payoff. We know such a network exists by Theorem 7 and the fact that there are only a finite number of possible networks. If (a)-(d) in the paragraph preceding the theorem are satisfied, we are done. If not, (d) must be violated. No additional network can draw members from $\mathcal{N}_{1}$, since the latter has the highest possible payoff. Among the viable networks that can draw members from the anonymous pool, let $\mathcal{N}_{2}$ be the one with the highest payoff. If (a)-(d) are now satisfied, we are done. Otherwise only (d) can be violated. We repeat the process until (d) is no longer violated.

\section{Conclusion}

Networks have properties that allow them to persist in a market economy despite their relative inability to exploit economies of scale and the other efficiency-enhancing properties of markets. Among these properties, and the one explored in this paper, is the capacity of networks to support enforcement of prosocial behavior among network members. Networks have this capacity by virtue of their ability to reduce information costs, thus permitting the emergence of 'trusting' Nash equilibria that do not exist, or are unstable, when information costs are high. Our particular model of these relationships could readily be extended to capture other salient aspects of the determinants of network formation, parochial exclusion, and network extinction. For example, because parochialism makes networks not only smaller, but more homogeneous as well, corresponding efficiency-enhancing effects of similarity or social affinity with parochial networks may be important.

The value of the informal contractual enforcement capacities of networks, the viability of networks, the range of viable network sizes, and the range of feasible degrees of parochialism all depend importantly on the nature of the goods and services that make up economic exchanges. Kollock (1994, p. 341) investigated "the structural origins of trust in a system of exchange" using an experimental design based on the exchange of goods of variable quality. He found that trust in and commitment to trading partners as well as a concern for one's own and others' reputations emerges when product quality is variable and non-contractible but not when it is contractible. These experimental results appear to capture some of the structure of actual exchanges. Siamwalla's (1978) study of marketing structures in Thailand contrasts the impersonal structure of the wholesale rice market, where the quality of the product is readily assayed by the buyer, with the personalized exchange based on trust in the raw rubber market, where quality is impossible to determine at the point of purchase. Thus, were technologies to evolve such that quality and quantity of the goods being transacted are readily subject to complete contracting, preferential trading within networks would be of little benefit and would likely be extinguished due to the implied foregone gains from trade. Conversely, were the economy to evolve in ways that heighten the problem of incomplete contracting we would expect to see growing economic importance of networks.

Applying this reasoning to our model, we consider the latter more likely. As production shifts from goods to services, and within services to information-related services (Quah, 1996), and as team-based production methods increase in importance, the gains from cooperation will increase as well because such activities involve relatively high monitoring costs and are subject to costly forms of opportunism. If this is the case the benefits associated 
with the mutual defect payoff (c) relative to the mutual cooperate payoff (b) will decline over time. ${ }^{15}$ Further, advances in communications technology arguably increase the number $(\kappa)$ of acquaintances from whom we can gather information at limited cost as well as increase the intelligibility of messages, especially across cultural, linguistic, or other group boundaries. The result would be to enhance the signal quality $(p)$ for a given degree of parochialism and hence level of network heterogeneity. The following are consequences.

The following are consequences. First, differentiating (13) we find that an increase in $b, \kappa$ or $\tau$, or a decrease in $c$, raises the payoff $\pi^{*}$ to network members in a trust equilibrium for a given size of network, thus making network membership more attractive relative to trading in the anonymous pool. Second, differentiating (17) with respect to the same variables we find that the effects of $\kappa, \tau, b, c$, and $x_{\max }$ are of the same sign as for $\pi^{*}$. Thus, better communication or higher payoffs to mutual cooperation relative to mutual defection in the network will increase the largest network size at which the signal quality will support a trust equilibrium. Third, because $\pi^{*}(x(\rho), \rho)$ is increasing in $x$ at $x_{\min }$, the upward shift in the $\pi^{*}$ function reduces $x_{\min }$. The intuition behind this result is that the increase in equilibrium payoffs in the trust equilibrium for a given size allows a network to bear increased costs of forgone trading opportunities occasioned by smaller size without becoming unviable.

As a result potential networks characterized by ascriptive filters that would in the past have resulted in groups either too large or too small to sustain a trust equilibrium may become viable as contracts become more costly to express completely and to enforce and as communication improves, and the payoffs to existing parochial networks may rise relative to the payoffs among anonymous traders.

On the other hand the kinds of social exclusion motivating network-based parochialism often violate strongly held universalistic norms and may encounter legal prohibition or other public policies motivated by a positive valuation of both tolerance and diversity of social interactions.

\section{Acknowledgements}

We are grateful to Katherine Baird, Roland Bénabou, Robert Boyd, Colin Camerer, Jeffrey Carpenter, Vincent Crawford, Steven Durlauf, Marcus Feldman, Edward Glaeser, Avner Greif, David Laibson, Michael Macy, Paul Malherbe, Jane Mansbridge, Corinna Noelke, Paul Romer, Barkley Rosser, Leigh Tesfatsion, Martin Weitzman, Peyton Young, participants in seminars at the NBER Summer Institute, The Santa Fe Institute, The University of

\footnotetext{
15 An increase in the cooperative payoff $b$ does not make the standard prisoner's dilemma interaction any 'easier to solve' of course, but it may enhance evolutionary pressures for the emergence of new rules of interaction that effectively mitigate the dilemma. Wade (1988, p. 774-775) describes such a process:

a significant number of the villages (in one small part of Upland South India) have institutions for the provision of public goods and services, which are autonomous of outside agencies in origin and operation. ... Only a few miles may separate a village with a substantial amount of corporate organization from others with none... Why the differences between villages? It is not because of differences in norms or values, for the villages are located within a small enough area for the culture to be uniform. It is rather because of differences in net collective benefit.
} 
Siena, and Yale University for perceptive comments, and to the John D. and Catherine T. MacArthur Foundation and The Santa Fe Institute for financial support.

\section{Appendix A. Proofs}

Proof of Theorem 3. At the marketplace for good $i$, the number $\xi_{i}$ of buyers and the number $\chi_{i}$ of sellers are independently distributed binomial random variables with means $x_{i} \psi_{i}(\rho)$ and $x_{i}\left(1-\psi_{i}(\rho)\right)$, respectively, and variance $x_{i} \psi_{i}(\rho)\left(1-\psi_{i}(\rho)\right) / 2$. The expected number of agents not finding a trade is thus $E\left[\left|\xi_{i}-\chi_{i}\right|\right]$, where the expectation is with respect to the product distribution. Consider a single marketplace, and let $\psi=\psi_{i}(\rho)$ be the probability an agent is a buyer, given level of parochialism $\rho$. Let $\tilde{x}$ be a random variable that takes the value 1 with probability $\psi$ and -1 with probability $1-\psi$. The sum of $x$ independent random variables distributed according to $\tilde{x}$ has expected value $x(2 \psi-1)$ and variance $4 x p(1-\psi)$. We assume $x(\rho)$ large enough relative to $k$ that the normal approximation to the binomial is sufficiently accurate ( $x>10$ is enough to ensure this). The excess number of buyers is thus distributed as a normal variate with mean $x(2 \psi-1)$ and variance $4 x p(1-\psi)$. It is easy to check that the probability of obtaining a trade is given by

$$
q(x, \psi)=1-\frac{g(x, \psi)}{x},
$$

where

$$
g(x, \psi)=4 \mathrm{e}^{-(1-2 \psi)^{2} x / 8 \psi(1-\psi)} \sqrt{\frac{\psi(1-\psi) x}{2 \pi}}+(2 \psi-1) x \operatorname{erf}\left[\frac{(2 \psi-1) x}{\sqrt{8 \psi(1-\psi) x}}\right]
$$

and $\operatorname{erf}[y]=(2 / \sqrt{\pi}) \int_{0}^{y} \mathrm{e}^{-t^{2}} \mathrm{~d} t$. We then find that

$$
\frac{\partial q}{\partial x}=\frac{\mathrm{e}^{-(1-2 \psi)^{2} x / 8 \psi(1-\psi)} \psi(1-\psi)}{\sqrt{\pi \psi(1-\psi) x / 2}(x-g(x, \psi))},
$$

which is strictly positive. Thus $q(x, \psi)$ is increasing in $x$. Since $x=x(\rho)$ is a decreasing function of $\rho, q$ is a decreasing function of $\rho$ via its first argument.

The expression for $\partial q / \partial \psi$ is complicated, but has the opposite sign of

$$
2 \mathrm{e}^{(1-2 \psi)^{2} x / 8 \psi(1-\psi)} \operatorname{erf}\left[\frac{(2 \psi-1) x}{\sqrt{8 \psi(1-\psi) x}}\right]+\frac{(1-2 \psi) \sqrt{2}}{\sqrt{\pi \psi(1-\psi) x}} .
$$

By expanding the integral in the erf function, we can show that this expression has the same sign as $(2 \psi-1)$ for $x \geq 1$. Thus if increased parochialism increases the average disparity between buyers and sellers in market $i, q$ is a decreasing function of $\rho$ via its second argument as well.

\section{References}

Acheson, J., 1988. The Lobster Gangs of Maine. New England Universities Press, Hanover, NH.

Adams, R., 1974. Anthropological perspectives in ancient trade. Current Anthropology 15, 239-258. 
Akerlof, G.A., 1997. Social distance and social decisions. Econometrica 65 (5), 1005-1027.

Baker, W.E., 1984. The social structure of a national securities market. American Journal of Sociology 89 (4), $775-811$.

Banerjee, A.V., Besley, T., Guinnane, T.W., 1994. Thy neighbor's keeper: the design of a credit cooperative with theory and a test. Quarterly Journal of Economics 109 (2), 491-515.

Barr, A., 2001. Social Dilemmas, Shame Based Sanctions, and Shamelessness: Experimental Results from Rural Zimbabwe. Working Paper, Oxford University.

Ben-Porath, Y., 1980. The F-connection: families, friends, and firms and the organization of exchange. Population and Development Review 6 (1), 1-30.

Bénabou, R., 1996. Heterogeneity, stratification and growth: macroeconomic implications of community structure and school finance. American Economic Review 86, 584-609.

Bernstein, L., 1992. Opting out of the legal system: extralegal contractual relations in the diamond industry. Journal of Legal Studies 21 (1), 115-158.

Berscheid, E., Walster, E., 1969. Interpersonal Attraction. Addison-Wesley, Reading, MA.

Blau, P., 1964. Exchange and Power in Social Life. Wiley, New York.

Bowles, S., Gintis, H., 1998. The moral economy of community: structured populations and the evolution of prosocial norms. Evolution \& Human Behavior 19 (1), 3-25.

Bowles, S., Gintis, H., 2002. Social capital and community governance. Economic Journal 112 (483), 419436.

Cohen, J.M., 1977. Sources of peer group homogeneity. Sociology of Education 15 (4), 227-241.

Craig, B., Pencavel, J., 1992. The behavior of worker cooperatives: the plywood companies of the Pacific Northwest. American Economic Review 82 (5), 1083-1105.

Craig, B., Pencavel, J., 1995. Participation and productivity: a comparison of worker cooperatives and conventional firms in the plywood industry. Brookings Papers: Microeconomics, 121-160.

Durlauf, S., 1996. Neighborhood feedbacks, endogenous stratification, and income inequality. Dynamic Disequilibrium Modelling. Cambridge University Press, Cambridge, UK, pp. 505-534.

Fafchamps, M., Minten, B., 2001. Returns to Social Capital Among Traders. Oxford University Working Paper.

Fehr, E., Gächter, S., 2002. Altruistic punishment in humans. Nature 415, 137-140.

Gellner, E., 1985. Nations and Nationalism. Cornell University Press, Ithaca.

Gintis, H., 2000. Game Theory Evolving. Princeton University Press, Princeton, NJ.

Glaeser, E.L., 2000. Cities and ethics: an essay for Jane Jacobs. Journal of Urban Affairs 22 (4), 473-493.

Granovetter, M., 1985. Economic action and social structure: the problem of embeddedness. American Journal of Sociology 91 (3), 481-510.

Greif, A., 1994. Cultural beliefs and the organization of society: an historical and theoretical reflection on collectivist and individualist societies. Journal of Political Economy 102 (5), 912-950.

Hechter, M., 1990. The attainment of solidarity in intentional communities. Rationality and Society 2(2), 142-155.

Hofbauer, J., Sigmund, K., 1998. Evolutionary Games and Population Dynamics. Cambridge University Press, Cambridge, UK.

Hollander, H., 1990. A social exchange approach to voluntary cooperation. American Economic Review 80 (5), 1157-1167.

Homans, G., 1961. Social Behavior: Its Elementary Forms. Harcourt Brace, New York.

Homans, G.C., 1958. Social behavior as exchange. American Journal of Sociology 65 (6), 597-606.

Iannaccone, L.R., 1992. Sacrifice and stigma: reducing free-riding in cults, communes, and other collectives. Journal of Political Economy 100 (2).

Kandel, D., 1978. Homophily, selection and socialization in adolescent friendships. American Journal of Sociology 84 (2), 427-436.

Kandori, M., 1992. The use of information in repeated games with imperfect monitoring. Review of Economic Studies 59 (3), 581-593.

Kaufman, J., 1995. How Cambodians came to control California doughnuts. Wall Street Journal, 1 and A14.

Kollock, P., 1994. The emergence of exchange structures: an experimental study of uncertainty, commitment, and trust. American Journal of Sociology 100 (2), 313-345.

Kotkin, J., 1993. Tribes: How Race, Religion and Identity Determine Success in the New Global Economy. Random House, New York. 
Kramer, R., Brewer, M., 1984. Effects of group identity on resource use in a simulated commons dilemma. Journal of Personality and Social Psychology 46 (5), 1044-1057.

Kranton, R., 1996. Reciprocal exchange: a self-sustaining system. American Economic Review 86 (4), 830851.

Kraybill, D.B., 1989. The Riddle of Amish Culture. Johns Hopkins Press, Baltimore.

Lawler, E.J., 1973. Motivation in Work Organizations. Brooks-Cole, Monterey.

Lazarsfeld, P.F., Merton, R.K., 1954. Friendship as a social process. In: Berger, M., et al. (Eds.), Freedom and Control in Modern Society. Van Nostrand, Princeton.

Longhofer, S., 1996. Cultural affinity and mortgage discrimination. Economic Review of the Federal Reserve Bank of Cleveland 32 (3), 12-24.

Loury, G., 2001. The Anatomy of Racial Inequality. Harvard University Press, Cambridge, MA.

Masclet, D., Noussair, C., Tucker, S., Villeval, M.-C., 2003. Monetary and nonmonetary punishment in the voluntary contributions mechanism. American Economic Review 93 (1), 366-380.

McElreath, R., Boyd, R., Richerson, P., 2003. Shared norms and the evolution of ethnic markers. Current Anthropology 44 (1), 122-129.

McMillan, J., Woodruff, C., 1999. Interfirm relationships and informal credit in Vietnam. Quarterly Journal of Economics 114 (4), 1285-1320.

Obot, I., 1988. Value systems in cross cultural contact: the effect of perceived similarity and stability on social evaluation. International Journal of Intercultural Relations 12, 363-379.

Ostrom, E., 1990. Governing the Commons: The Evolution of Institutions for Collective Action. Cambridge University Press, Cambridge, UK.

Pagano, U., 1995. Can economics explain nationalism? In: Breton, A., Galeotti, G., Salmon, P., Wintrobe, R. (Eds.), Nationalism and Rationality. Cambridge University Press, Cambridge, pp. 173-203.

Perko, L., 1991. Differential Equations and Dynamical Systems. Springer-Verlag, New York.

Platteau, J.-P., Seki, E., 2001. Community arrangements to overcome market failure: pooling groups in Japanese fisheries. In: Hayami, M., Hayami, Y. (Eds.), Communities and Markets in Economic Development. Oxford University Press, Oxford, pp. 344-402.

Quah, D., 1996. The Invisible Hand and the Weightless Economy, 1996. Occasional Paper. Centre for Economic Performance, London School of Economics.

Rauch, J.E., 1996. Trade and Networks: An Application to Minority Retail Entrepreneurship, June 1996. Russell Sage Working Paper.

Sahlins, M., 1972. Stone Age Economics. Aldine Press, Chicago.

Sampson, R.J., Raudenbush, S.W., Earls, F., 1997. Neighborhoods and violent crime: a multilevel study of collective efficacy. Science 277, 918-924.

Siamwalla, A., 1978. Farmers and middlemen: aspects of agricultural marketing in Thailand. Economic Bulletin for Asia and the Pacific 39 (1), 38-50.

Sugden, R., 1986. The Economics of Rights, Co-operation and Welfare. Basil Blackwell, Oxford.

Tajfel, H., Billig, M., Bundy, R.P., Flament, C., 1971. Social categorization and intergroup behavior. European Journal of Social Psychology 1, 149-177.

Taylor, C., 1997. The old boy network and the young-gun effect. International Economic Review 41 (4), 871891.

Taylor, P., Jonker, L., 1978. Evolutionarily stable strategies and game dynamics. Mathematical Biosciences 40, $145-156$

Thibaut, J., Kelly, H., 1959. The Social Psychology of Groups. Wiley, New York.

Udry, C., 1993. Credit markets in northern Nigeria: credit as insurance in a rural economy. In: Hoff, K., Braverman, A., Stiglitz, J.E. (Eds.), The Economics of Rural Organization: Theory, Practice, and Policy. Oxford University Press, New York.

Wade, R., 1988. Village Republics: Economic Conditions for Collective Action in South India. Cambridge University Press, Cambridge, UK.

Wade, R., 1988. Why some Indian villages cooperate. Economic and Political Weekly 33, 773-776.

Weber, E., 1976. Peasants into Frenchmen: The Modernization of Rural France, 1870-1914. Stanford University Press, Stanford.

Whyte, W.F., 1955. Money and Motivation. Harper \& Row, New York. 
Wilson, D.S., Sober, E., 1994. Reintroducing group selection to the human behavioral sciences. Behavior and Brain Sciences 17, 585-654.

Wintrobe, R., 1995. Some economics of ethnic capital formation conflict. In: Breton, A., Galeotti, G., Salmon, P., Wintrobe, R. (Eds.), Nationalism and Rationality. Cambridge University Press, Cambridge, pp. 43-70.

Woodyard, C., 1995. Roadside revival by Patels. Los Angeles Times 1, A40-A41.

Yamagishi, T., 2003. The group heuristic: a psychological mechanism that creates a self-sustaining system of generalized exchanges. In: Proceedings of the Santa Fe Institute Workshop on the Coevolution of Institutions and Behavior. 